\title{
Optical Probing of Single Fluorescent Molecules and Proteins
}

\author{
María F. García-Parajó, ${ }^{*[a]}$ Joost-A. Veerman, ${ }^{[a]}$ Rudo Bouwhuis, ${ }^{[a]}$ Renaud Vallée, ${ }^{[a, b]}$ \\ and Niek F. van Hulst ${ }^{[a]}$
}

Single-molecule detection and analysis of organic fluorescent molecules and proteins are presented, with emphasis on the underlying principles, methodology and the application of singlemolecule analysis at room temperature. This Minireview is mainly focused on the application of confocal and near-field optical microscopy to investigate the photodynamics of individual molecules embedded in ultrathin polymer layers. We discuss rotational mobility of individual probe molecules in polystyrene and poly(methylmethacrylate) thin films, fluorescence lifetime trajectories and their spatial distribution, and real-time singlettriplet dynamics. As a whole, the single-molecule photodynamics observed is due to the dynamic nature of both polymers at room temperature, where local polymer conformational dynamics modulates the oxygen concentration and diffusion on a molecular scale, influencing the fluorescence lifetime and intersystem crossing parameters. We also discuss the photodynamics of individual autofluorescent proteins, in particular the on/off blinking and the apparent stability of the protein against bleaching. These studies illustrate the unique information obtainable with the singlemolecule approach, information that is otherwise hidden in ensemble-averaged measurements.

\section{KEYWORDS:}

blinking - green fluorescent protein - near-field scanning optical microscopy · quantum jumps • single-molecule microscopy

\section{Introduction}

Looking at only one molecule at a time has long been the dream of many scientists. In every textbook on physics, chemistry or biology, individual molecules are used to explain basic principles, such as the interaction of light with matter, chemical reactions or cellular processes in living organisms. However, drawing a single molecule on paper is much easier that observing one in reality! Until about ten years ago, all our scientific knowledge was based on observations of the average behaviour of large ensembles of molecules, just because individual molecules could not be distinguished from the ensemble.

Within the last decade however, technological barriers for observing single molecules have been overcome by instrumentational innovations in microscopy. Different techniques have been developed for single-molecule detection (SMD), each of them probing distinct molecular properties. For example, molecules can be "felt" by very sharp probes that measure forces (atomic force microscopy, AFM) ${ }^{[1]}$ or electric charges (scanning tunnelling microscopy, STM). ${ }^{[2]}$ In 1993, near-field scanning optical microscopy (NSOM) was used for the first observation of single fluorescent molecules at room temperature. ${ }^{[3]}$ Optical detection of a single fluorescent molecule literally involves "looking at" the molecule while it emits light. The focus of this Minireview is the optical probing of single fluorescent molecules and autofluorescent proteins in complex environments at room temperature.

Why is it useful to study individual molecules in a complex environment? Actually, there are distinct advantages of single- molecule measurements over ensemble-averaged experiments. A single-molecule experiment reveals the full distribution of a given molecular property, while an ensemble measurement only yields a statistical average. Thus, static and dynamic heterogeneity in a population of molecules in a complex condensed system can be identified and related to the molecular environment. Moreover, in systems under equilibrium conditions, singlemolecule time trajectories exhibit stochastic fluctuations that directly contain detailed statistical and dynamical information. Time-dependent processes and reaction pathways in nonequilibrated systems can be followed and studied without the need for synchronising a population of molecules. Finally, singlemolecule detection provides information over the structure and function of molecules that is difficult or impossible to obtain

[a] Dr. M. F. García-Parajó, Dr. J.-A. Veerman, R. Bouwhuis, Dr. R. Vallée, Prof. Dr. N. F. van Hulst

Applied Optics Group

Faculty of Applied Physics \& MESA ${ }^{+}$Research Institute University of Twente

P.O. Box 217, 7500 AE Enschede (The Netherlands)

Fax: (+31) 53-489 1105

E-mail:M.F.garciaparajo@tn.utwente.nl

[b] Dr. R. Vallée

Polymer Science Group

Faculty of Chemical Technology \& MESA ${ }^{+}$Research Institute University of Twente

P.O. Box 217, 7500 AE Enschede (The Netherlands) 
using averaging techniques. For instance, rare photophysical and photochemical processes otherwise hidden in the ensemble can be uncovered and studied in detail.

Single-molecule studies at room temperature have allowed monitoring of the time development of various observables. For example, translational, ${ }^{[4-8]}$ rotational, ${ }^{[7,9-11]}$ spectral ${ }^{[12-16]}$ and enzymatic-turnover ${ }^{[17]}$ trajectories showing dynamic behaviour on a typical time scale of milliseconds to tens of seconds have been recorded. Excellent reviews on these issues have been published recently. ${ }^{[18-20]}$ Such experiments are useful to probe, for example, membrane transport mechanisms in biological systems ${ }^{[4]}$ or conformational changes of macromolecules, including chemical polymers $^{[21-23]}$ and biological ones such as proteins ${ }^{[17,24]}$ or DNA. ${ }^{[24,25]}$ Conformational motion of biological macromolecules is being studied on a single-molecule basis by monitoring the fluorescence lifetimes ${ }^{[26,27]}$ or by single-pair fluorescence resonance energy transfer (spFRET) experiments. ${ }^{[28]}$ In short, singlemolecule techniques have rapidly expanded to include a wide range of life sciences. Experiments on molecular motors, DNA transcription, enzymatic reactions, protein dynamics and cell signalling have been summarised in recent reviews. ${ }^{[29-31]}$ Finally, multichromophoric systems, such as aggregates and conjugated polymers, are receiving increasing attention because of excitonic (de)localisation, which makes that these systems behave as a single quantum system displaying discrete on/off switching. ${ }^{[22,32-34]}$

In this Minireview, the requirements and methodology used for the optical detection of individual molecules at room temperature will be discussed. We focus mainly on the application of confocal and near-field optical microscopy to investigate the photodynamics of individual molecules embedded in thin polymer films. Single-molecule detection methods are exploited to gain information about the nanoenvironment of the molecules, namely the intrinsic properties of the thin polymer films used in the experiments. This is achieved by monitoring translational and rotational mobility by means of fluorescence lifetime trajectories and real-time singlet-triplet dynamics of individual molecules embedded in different types of the polymer matrices. We also discuss the photodynamics of individual autofluorescent proteins embedded in poly(acrylamide) gels and show in here how single-molecule analysis can be used as groundwork for applications in life sciences and in vivo experiments at the single-molecular level.

\section{Single-Molecule Detection Methodology and Analysis}

\subsection{General Principles}

The light absorption and emission (fluorescence) of a typical dye molecule can be described in terms of the Jablonski diagram, ${ }^{[35]}$ as depicted in Figure 1. After absorption of a photon, a molecule exists in an excited state and, because it is not in thermal equilibrium with its surroundings, it will in general have only a short lifetime, since a number of processes will contribute to the deactivation of the excited molecule to the lower state. After fast

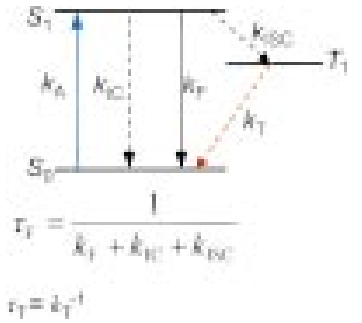

Figure 1. Three-level scheme showing absorption from the lowest excited singlet state $S_{0}$ to the first excited state $S_{1}$ (with rate constant $k_{A}$ ), fluorescence emission from $S_{1}$ to $S_{0}\left(k_{F}\right)$, internal conversion from $S_{1}$ to $S_{0}\left(k_{1}\right)$, intersystem crossing from $S_{1}$ to the lowest excited triplet state $T_{1}\left(\mathrm{k}_{15 \mathrm{C}}\right)$ and transition from $T_{1}$ to $S_{0}\left(\mathrm{k}_{T}\right)$. The triplet lifetime $\tau_{T}$ is much longer $\left(\sim 10^{5}\right)$ than the fluorescence lifetime $\tau_{F}$, and the intersystem crossing rate is much smaller $\left(\sim 10^{4}\right)$ than the fluorescence rate.

vibrational relaxation the molecule can return to the groundstate $S_{0}$ by emitting a fluorescence photon. The average period of time that the molecule spends in the exited state $S_{1}$, the fluorescence lifetime $\tau_{\mathrm{F}}$, is in order of a few nanoseconds for most organic dyes. This value actually sets the maximum emission rate of a molecule at $\sim 10^{8} \mathrm{~s}^{-1}$ for high excitation intensity, that is at optical saturation. A molecule in the $S_{1}$ state can also undergo conversion to the first triplet state $T_{1}$. Intersystem crossing (ISC) from $S_{1}$ to $T_{1}$ is spin forbidden, as well as the transition from $T_{1}$ to the ground-state $S_{0}$. As a result, the rate constant $k_{\mathrm{ISC}}$ for such events to occur is several orders of magnitude smaller than that of fluorescence, and the lifetime of the triplet-state $\tau_{\top}$, typically from in the range of microseconds to seconds, is much longer than $\tau_{\mathrm{F}}$.

Two main facts have to be taken into account when detecting single molecules at room temperature. Firstly, the absorption cross-section of typical dyes is orders of magnitude smaller at room temperature $\left(\sim 10^{-16} \mathrm{~cm}^{2}\right)$ as compared to cryogenic temperatures $\left(\sim 10^{-10} \mathrm{~cm}^{2}\right) .{ }^{[20]}$ As a result, the "capability" of a molecule to absorb a photon is greatly reduced at room temperature. A second important phenomenon occurring at ambient temperature is photobleaching, that is, a photochemical reaction of the molecule while in the excited state, which irreversibly alters its chemical structure. At room temperature, photobleaching occurs after emission of roughly $10^{6}$ photons, limiting in practice the observation of a single molecule to a few seconds.

The main difficulty in single-molecule detection is to discriminate the fluorescence of a single molecule amongst millions of other molecules also contained in the medium (liquid or condensed phase). Essentially two requirements must be met in order to detect the fluorescence arising from a single molecule. First, the signal from the single molecule must be larger than that of any interfering background signal. This can be accomplished by reducing the background and by optimising the fluorescence collection efficiency. Secondly, the concentration of molecules in the sample must be small enough to guarantee that only one molecule at the time is being excited. The most common techniques to efficiently detect individual fluorescent molecules at room temperature are described in the next Section. 


\subsection{Methods}

The requirements of severe background reduction, high detection efficiency and spatially selective imaging necessary for single-molecule detection are achieved using near-field microscopy, as well as far-field methods such as confocal, bright-field and total internal reflection (dark-field) microscopy. Figure 2 shows these techniques schematically. Lately, two-photon fluorescence detection, ${ }^{[36-38]}$ single-molecule excitation with surface plasmons on metal surfaces ${ }^{[39]}$ and surface-enhanced Raman spectroscopy on single molecules ${ }^{[40,41]}$ have been reported.

Since the background cross-sections are proportional to the excitation volume, most techniques developed for singlemolecule detection are based on a reduced excitation volume. By far the smallest excitation volume is provided by near-field microscopy (Figure $2 \mathrm{a}$ ), which strongly reduces sample background and results in a large spatial selectivity (approximately $\left.10^{5} \mathrm{~nm}^{3}\right)$. In contrast, the diffraction-limited excitation volume in confocal microscopy (Figure $2 \mathrm{~b}$ ) is more than three orders of magnitude larger (typically $10^{8} \mathrm{~nm}^{3}$ ), which imposes restrictions on the minimal distance between two adjacent molecules to about $0.5 \mu \mathrm{m}$ at best and increases the amount of unwanted background. On the other hand, confocal excitation has the advantage of a less-complex instrumentation and more practical freedom to implement sample chambers for, among others, liquid immersion or pressure control. In the detection path of both Figures $2 \mathrm{a}$ and $2 \mathrm{~b}$ a photon-counting detector (usually an avalanche photodiode, APD) is confocally aligned with the excitation volume. For imaging, the sample must be scanned a)

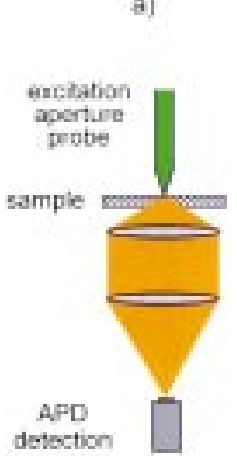

b)

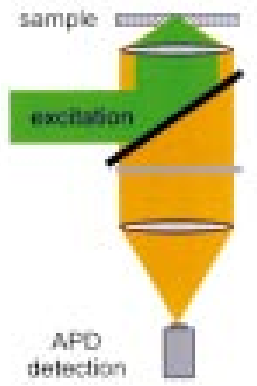

c)

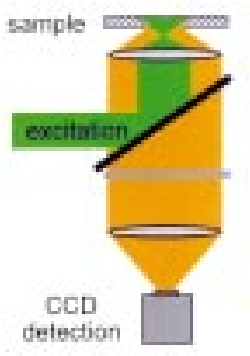

d

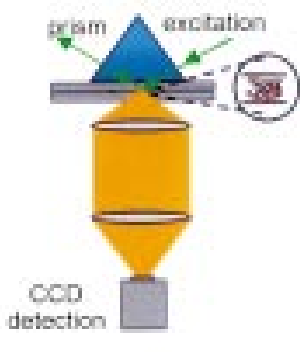

Figure 2. Four experimental approaches to detect single molecules: a) near-field microscopy, b) confocal microscopy, c) wide-field microscopy and d) total internal reflection (dark-field) microscopy. The requirements for single-molecule detection with regard to background reduction, high detection sensitivity and spatially selective imaging are fulfilled in a different way for each of the four configurations, which results in particular benefits and disadvantages for each method. Near-field microscopy has the smallest illumination volume resulting from subwavelength illumination through a small aperture optical-fibre probe. A superior lateral optical response of $\sim 70 \mathrm{~nm}$ on a single molecule can be obtained, which results in localisation accuracy down to only a few nanometres. ${ }^{[49]}$ The small excitation volume also allows independent observation of more closely packed molecules, typically $\sim 100$ molecules $\mu m^{-2}$ on a surface. As result of the evanescent characteristic of the near-field excitation, only molecules within the first few tenths of nanometers away from the surface are efficiently excited, which is extremely useful for the detection of individual molecules on real cell membranes. However, technological obstacles concerning the fabrication of the probe itself, its low throughput, the need of a sample-to-probe distance regulation, as well as the complicating presence of metallic probe in close proximity to the molecule, have directed current single-molecule research towards more flexible, far-field methods, such as confocal and wide-field microscopy. In particular, applying total internal reflection and widefield approaches, a large area of the sample can be illuminated, which allows simultaneous detection of several individual molecules using a CCD camera. through the excitation volume, which should contain no more than one molecule at the time. This is an important difference between bright-field (Figure $2 \mathrm{c}$ ) and dark-field (Figure $2 \mathrm{~d}$ )

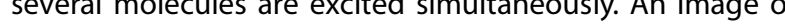
array (usually a charged coupled device, CCD camera). drawback of bright-field microscopy is the large excitation volume compared to confocal or near-field microscopy, resulting in a large amount of background. This problem can be cicumvented in dark-field microscopy, where the axial depth evanescence fied formed at a refactive internal reflection geometry (Figure $2 \mathrm{~d}$ ). Dark- and bright-field approaches are particularly suited to investigate the behaviour of a large number of molecules at the same time but have the of the CCD camera) as compared to confocal or near-field methods.

\section{Overview of Results}

\subsection{Photophysics of Individual Dyes in Polymers}

mers are long-chain molecules of very high molecular shape and organisation of the crystallites depend on how the ${ }^{[42]}$ Other polymers are amorphous, often because their chains are too irregular to permit ordered packing. The onset of chain molecular motion heralds the glass transition and softening of the polymer from the glassy to the rubbery state. Microstructural aspects of polymer glasses have been considered for a long time. ${ }^{[43]}$ There is strong evidence that in the glassy phase the chain microstructure is essentially statistical in nature. ${ }^{[44]}$ In contrast, the possibility of having a short-range molecular order (that is, local chain folding) has been raised by many researchers. ${ }^{[45]} \mathrm{De}$ spite intensive research, the issue of the microstructure of macromolecules in amorphous polymers remains largely unsettled.

If a polymer is cooled slowly to absolute zero, it will achieve its closest packing. However, because of the large macroscopic viscosity of the solid, it seldom achieves an ultimate packing density in finite times and hence, even at absolute zero, a polymer matrix will contain some "free volume". ${ }^{[42,43]}$ At room temperature, thermal motion will expand the solid, which in turn will create additional free 
volume allowing the diffusion of small-molecule reagents, such as oxygen. ${ }^{[46]}$ One important aspect of the free volume is that it can confer upon the amorphous polymer a large mobility even after vitrification (in, for example, an apparently rigid solid) as it allows localised molecular motions in the glass to occur. These will ultimately give rise to relaxation processes which determine the physical properties of the polymer.

Amongst the many different techniques developed in the last 40 years to investigate polymer microstructure and dynamics, static and dynamic fluorescence spectroscopy of probe molecules dissolved in or linked to polymer chains plays a major role. ${ }^{[46,47]}$ When the fluorescence spectrum and/or decay dynamics of the excited molecular states are sensitive to the polarity of their local environment, they can provide useful information about the polarity of occupied sites in homogeneous or microheterogeneous systems. Fluorescence decay profiles have shown to be especially valuable in discerning the type and number of environments in which the probe molecule resides. ${ }^{[4]}$ However, all the measurements reported so far are obtained by collecting the fluorescence from a large volume of the polymer containing many fluorescence probes. Single-molecule fluorescence detection has the potential of delivering information on the nanoheterogeneity of the environment, nanostructure of the bulk and surface of glassy polymers and ascertaining with nanometer accuracy different regions where crystalline and amorphous phases might coexist. A summary of the research performed on individual molecules embedded in thin amorphous polymer matrices is discussed below. As an emerging field, the examples shown here are meant to illustrate the potential of single-molecule detection to provide unique information on polymer molecular motion, polarity and diffusion of small molecules in polymers.

We concentrate here on two particular polymer systems, atactic poly(methyl methacrylate) and atactic polystyrene (PMMA and PS). The chemical structure of both polymers is shown in Scheme $1 \mathrm{a}$.
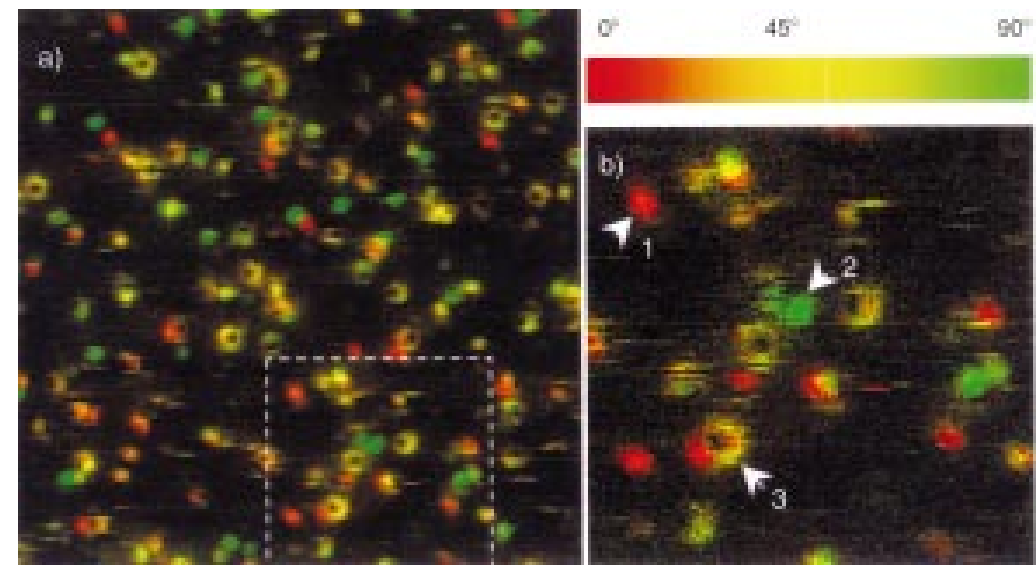

Figure 3. Near-field fluorescence image, obtained with a $70 \mathrm{~nm}$ diameter aperture probe, showing individual Dil molecules embedded in $a \sim 10 \mathrm{~nm}$ PMMA layer. a) The image corresponds to a scanning area of $4.8 \times 4.8 \mu \mathrm{m}^{2}$, with a pixel acquisition time of $2.5 \mathrm{~ms}$ and $8 \mathrm{nmpixel}^{-1}$. The background level in the image is about 4 counts pixel $^{-1}$ and the maximum signal is

60 counts pixel $^{-1}$. The colour in the image reflects the in-plane orientation of the molecules while the shape of the fluorescent spots is the result of the overlap of the absorption dipole moment of the molecules and the exciting electric field of the NSOM probe. b) Detailed picture from the region marked in (a). Molecules 1 and 2 have in-plane orientations close to $0^{\circ}$ and $90^{\circ}$, respectively, while molecule 3 has $a \sim 90^{\circ}$ out-of plane orientation. a)

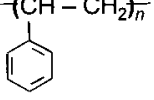

Polystyrene
Scheme 1. a) Structure of the polymer systems used in our studies: PMMA on the left and PS on the right side. b) Structure of Dil, in the example given here the 18member alkyl chain is further identified as DilC ${ }_{18}$.
Carbocyanine probe molecules, either 1,1'-dioctadecyl-3,3,3',3'-tetramethylindocarbocyanine perchlorate ("Dil", DilC $_{18}$; see Scheme 1 b) or 1, $1^{\prime}$-dioctadecyl-3,3,3',3'-tetramethylindodicarbocyanine perchlorate ("DiD" oil, $\mathrm{DilC}_{18}$ oil), have been dissolved at a low concentration $\left(10^{-8}-10^{-9} \mathrm{M}\right)$ in the polymer containing solution prior to film formation. In all the cases described here, ultrathin polymer layers have been prepared by spin coating at typically $3000 \mathrm{rpm}$. Prior to single-molecule experiments the samples have been inspected by AFM. Only samples with complete film formation and thickness of $\sim 10-$ $18 \mathrm{~nm}$ have been used.

\subsubsection{Single-Molecule Imaging}

Figure $3 \mathrm{a}$ shows a fluorescence image of several hundred individual Dil molecules embedded in a thin PMMA layer. The image has been obtained using circularly polarised excitation in the near-field configuration, that is subwavelength illumination through the small aperture of a near-field optical probe. The sample is scanned through the illumination volume and molecules are excited if the molecular absorption dipole moment matches the local field vector of the near-field aperture probe. The generated fluorescence is collected in the far field and the image is built up in time from the top left to the bottom right corner. Such an image is therefore both a time record as well as a spatial record of the signal. Polarisation-sensitive detection has been used while acquiring the image so that at each moment the relative intensities of the polarised fluorescence signals are recorded using two perpendicular channel detectors. ${ }^{[7]}$

The bright spots on the image correspond to the fluorescence emission of individual DilC $_{18}$ molecules. A pseudo-colour scale is used to indicate the polarisation direction of the fluorescence 
and thus the in-plane orientation of the emission dipole moment of the molecules. The colour scale ranges from green (for dipoles oriented along the vertical image direction) through yellow to red (for dipoles oriented in the horizontal image direction). The size of the fluorescent spot results from the convolution between the light emanating from the near-field probe and the radiating molecule. In this image, the spot size is $\sim 70 \mathrm{~nm}$, which indeed corresponds to the probe size diameter used in the experiments. In addition, the molecules exhibit different shapes in the image, which is caused by the overlap of the molecular absorption dipole moment with the different polarisation components of the electric field of the probe. ${ }^{[3,48,49]}$

It is clear from Figure 3 a that all the molecules have a defined polarisation emission (a characteristic and unique colour per spot). Figure $3 \mathrm{~b}$ shows a subset of the molecules in more detail. One important advantage of near-field microscopy over far-field methods is the direct possibility of imaging molecules oriented perpendicular to the sample plane..$^{[3,48,49]}$ This is because of the existence of near-field components in the direction perpendicular to the sample plane (the $z$-direction), which occur at the glass - metal interface of the near-field probe. ${ }^{[3,48,49]}$ The $z$-field mainly excites molecules with their absorption moment perpendicular to the sample surface. These molecules exhibit a ringshaped emission pattern, which is clearly visible for some molecules in Figures $3 \mathrm{a}$ and $3 \mathrm{~b}$. Thus, Figure $3 \mathrm{a}$ shows a large set of fluorescent molecules with a completely random 3D orientation in the PMMA matrix: Molecules with a ring shape mainly orient along the $z$-direction and molecules with a circular shape orient in the $x-y$ sample plane (red-green colour scale). A random orientation of the dyes is not surprising, since the film is completely amorphous and the probe molecules can take any spatial arrangement within the matrix.

Further observation of Figure 3 reveals no translational or rotational diffusion of the molecules over the time scale of the experiment (we have performed sequentially imaging over the same scan area over periods longer than one hour). This is confirmed by the fact that the fluorescence spots remain stationary in space and display the same colour coding during the scanning. Earlier observations on rhodamine (R6G) molecules dispersed on glass showed a translational diffusion of $D \sim 6.7 \times 10^{-15} \mathrm{~cm}^{2} \mathrm{~s}^{-1}$ and, when embedded in $25 \mathrm{~nm}$ thick poly(vinylbutyral) films, of $D \sim 2.6 \times 10^{-15} \mathrm{~cm}^{2} \mathrm{~s}^{-1}$. ${ }^{[6,7]}$ Slow rotational diffusion of dye molecules in polymer layers, on the time scale of several minutes, has been occasionally observed. ${ }^{[7,11]}$ Lateral and rotational diffusion of fluorescence-labelled lipids on supported phospholipid membranes has been also observed at the individual molecular level. Rotational and translational diffusion constants of $D_{\text {rot }}=7 \times 10^{7} \mathrm{rad}^{2} \mathrm{~s}^{-1}$ and $D_{\text {trans }}=3.5 \times$ $10^{-8} \mathrm{~cm}^{2} \mathrm{~s}^{-1}$, respectively, have been determined indicating significant mobility on the nanosecond time scale. ${ }^{[50]}$ In the case of the PMMA layer, translational and rotational motion is greatly reduced due to the large elastic modulus $\left(10^{10} \mathrm{Nm}^{-2}\right)$ of the polymer. ${ }^{[46]}$ However, because of the presence of free volume within the polymer, some rotational or translational movement of the probe molecules will be facilitated, especially for ultrathin films. This is rarely observed in our experiments and thus we conclude that the free volume contained in the PMMA film of
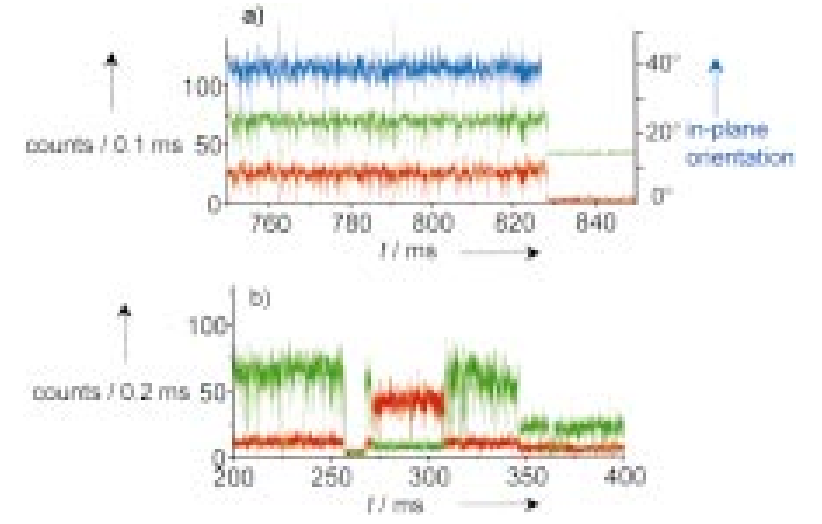

Figure 4. Time extracts of the fluorescence emission of two different Dil molecules embedded in PMMA. The total intensity emitted by the molecule is separated into two perpendicular components accounting for its relative in-plane orientation. The red signal corresponds to the fluorescence detected in the 0 channel, while the green signal corresponds to the $90^{\circ}$ channel. The total number of counts emitted by the molecule is the sum of the red and green channels. The fluorescence emission shows up as a constant signal with randomly occurring "dropouts" of the signal to the background level. The reason for these sudden interruptions of fluorescence emission is explained in detailed in Section 3.1.4 and Figure 9. a) The molecule displays stable emission and in-plane orientation. Note that the green signal has been shifted vertically for clarity. The blue signal corresponds to the calculated in-plane orientation of the molecule taking into account the relative contribution of both channels. At approximately $830 \mathrm{~ms}$ the molecule photodissociates, as seen by the sudden and permanent interruption of the fluorescence emission. b) The molecule exhibits rotational activity on a time scale of $\sim 50 \mathrm{~ms}$. Initially the molecule is oriented $\sim 70^{\circ}$. At $250 \mathrm{~ms}$, the molecule interrupts its emission for $\sim 10 \mathrm{~ms}$ and briefly recovers its emission with the same in-plane orientation. Only $2 \mathrm{~ms}$ later the molecule abruptly changes its orientation from $70^{\circ}$ to approximately $20^{\circ}$ and remains in this position for $\sim 40 \mathrm{~ms}$ after which a new rotation back to $70^{\circ}$ occurs. After $350 \mathrm{~ms}$, the molecule remains oriented around $70^{\circ}$ but with approximately half of its initial emission intensity.

thickness $\sim 10 \mathrm{~nm}$ is small enough to immobilise the probe molecules (with a size of $\sim 2 \mathrm{~nm}$ ) in space.

The observation of molecular diffusion in polymer hosts is important in view of the current discussions in the polymer community regarding the segment density in glassy polymer films near the film surface (or in thin films). There is growing evidence that in some polymers the top layer is much more mobile due to lower segment density at the surface. ${ }^{[51,52]}$ It has been postulated that even if the bulk is glassy (for example, PMMA at room temperature), the top thin layer $(5-10 \mathrm{~nm})$ or corresponding thin films might have an effective thin film glass transition temperature $T_{\mathrm{g}}$ which is below ambient temperature. ${ }^{[52]}$ Since above its $T_{\mathrm{g}}$ the material has a large degree of free volume, some motion is expected to occur which would undoubtedly manifest on the probe molecules embedded in the material. This is certainly not detected in our experiments, at least in the time scale of our observations where an area is monitored over $\sim 1$ hour.

Figure 4 a shows the time-trace trajectory of a single molecule with the relative intensity signals collected by both perpendicular detectors, as well as its relative in-plane orientation. Such a time trace is acquired by positioning the near-field probe directly above the molecule and monitoring the fluorescence continuously as a function of time. The in-plane orientation of the 
molecule can be easily determined by taking the arctangent of the square root of the ratio of the measured integrated intensities in the two $\left(0^{\circ}, 90^{\circ}\right)$ detection channels, taking into account the background counts in each channel. ${ }^{[7]}$ As shown in the time trace, the orientation of the molecule is constant in time. Figure $4 \mathrm{~b}$ on the contrary shows a molecule displaying some degree of rotational dynamics. Clearly the molecule exhibits discrete "jumps" between two well-defined orientations, while the total intensity remains essentially the same. The latter indicates that only orientational jumps occur without altering the conformation of the molecule, namely the absorption crosssection and/or fluorescence yield.

In our experiments we have observed that only $5 \%$ of the molecules embedded in PMMA show some rotational activity. In contrast, for similar probe molecules embedded in PS thin films (of similar thickness), rotational dynamics has been observed for as many as $40 \%$ of the molecules studied. ${ }^{[53]}$ This is consistent with earlier results revealing sufficient free volume in PS to allow observable motion of the phenyl rings at temperatures well below room temperature. ${ }^{[46,54]}$ More experiments are obviously necessary before drawing conclusions concerning the different structural properties of both PMMA and PS polymers, in particular, chain-segment density at the surface and $T_{\mathrm{g}}$ for both polymers at such extreme film thinness. In any case, it is clear that single-molecule analysis can provide very detailed information on the distinct structural properties of different polymer matrices.

\subsubsection{Fluorescence Lifetime Trajectories}

Dynamic fluorescence spectroscopy is a well-known technique used to obtain information about the microstructure of polymeric materials. ${ }^{[47]}$ In the most general case, the fluorescence decay of isolated molecules is described by a single exponential. However, for molecules embedded in a polymer matrix the decay is influenced by interactions between the fluorophores and the polymer. Because most polymers are nonuniform media in a sense that there is no long-range structural order, the measured fluorescence decay of molecules is the average value over a distribution of many different fluorescence lifetimes. ${ }^{[55,56]}$ While research continues in order to model and understand the complex behaviour of fluorophores in interacting, nonuniform media like polymers, ${ }^{[55,56]}$ a much more exquisite and direct manner to investigate the influence of the environment on the fluorescence lifetime of a molecule is simply by using singlemolecule detection methods.

Fluorescence lifetimes of single molecules at room temperature were first measured by time-correlated single-photon counting (TCSPC) using the near-field configuration ${ }^{[57,58]}$ and later with far-field microscopy. ${ }^{[13]}$ In these experiments, the fluorescence lifetimes were determined from all photon counts emitted by single molecules that had been positioned under the laser illumination. While the near-field method requires the proximity of a metallised tip to the molecule, which influences some of the properties of the molecule under study, far-field methods are less invasive. For instance, Macklin et al. ${ }^{[13]}$ reported fluorescence lifetimes of single molecules simultaneously with
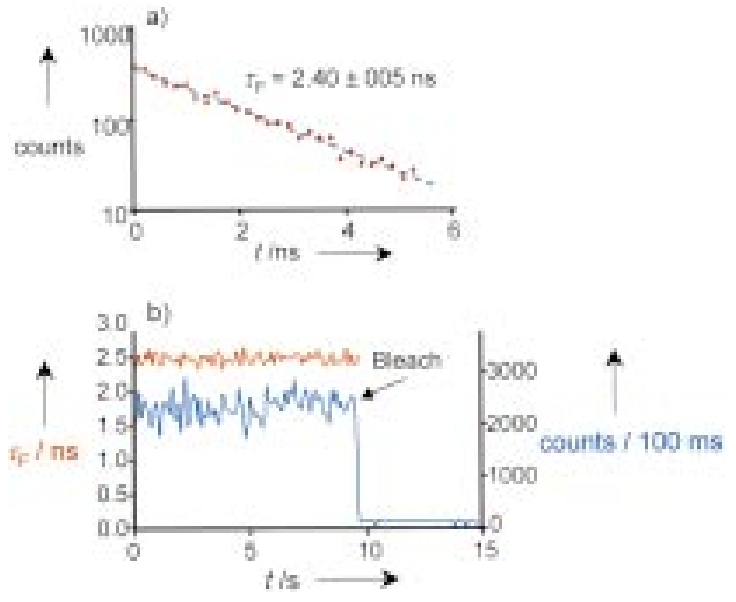

Figure 5. a) Histogram of the time differences between the excitation pulse and the fluorescence photon obtained with TCSPC. From the fluorescence decay histogram a characteristic lifetime of $\tau_{F}=2.4 \mathrm{~ns}$ for a particular molecule is obtained. b) Calculated fluorescence lifetimes $\tau_{F}$ (red line) binned into $100 \mathrm{~ms}$ time intervals and the corresponding fluorescence time trace (blue line) of the same molecule.

their emission spectra for molecules located at a polymer-air interface using confocal microscopy. Although the fluorescence lifetime decay measured for all the molecules displayed a single exponential behaviour, they reported a distribution of lifetime values with variations up to $50 \%$. Further experiments indicated that the different lifetimes measured were due to the relative orientation of the molecules with respect to the polymer-air interface. ${ }^{[13]}$ The workers gave no discussion as to the influence of the PMMA environment on the fluorescence decay of the molecules. Recently, Tinnefeld et al. reported a powerful method to monitor fluctuations in the excited lifetime of single molecules by recording fluorescence lifetime trajectories with millisecond time resolution. ${ }^{[59]}$ In agreement with the observations of Macklin et al., ${ }^{[13]} \mathrm{a}$ broad distribution of the fluorescence decay times for molecules dispersed in space was found and similarly attributed to the presence of a dielectric interface, which modifies the radiative component of the excited-state lifetime. We have done similar experiments with DiD molecules embedded in thin PMMA layers and have recorded temporal and spatial lifetime trajectories.

The fluorescence lifetime of a single molecule can be obtained by measuring the time lag between the exciting laser pulse and the fluorescence emission of the molecule. This time correlated single-photon experiment leads to a histogram containing all the delay times between consecutive excitation pulses and emitted photons. Figure 5 a shows such a log-scale histogram. From the slope of the histogram the fluorescence lifetime of this particular molecule is obtained. The histogram contains 3300 collected photocounts in an integration time of $100 \mathrm{~ms}$ and with a time resolution of 100 ps. Every $100 \mathrm{~ms}$ a new histogram can be built and, based on the fluorescence lifetimes extracted from consecutive histograms, both a fluorescence lifetime trajectory and a fluorescence intensity transient are created. Figure $5 \mathrm{~b}$ shows as an example the fluorescence lifetime trajectory and the fluorescence time trace for 


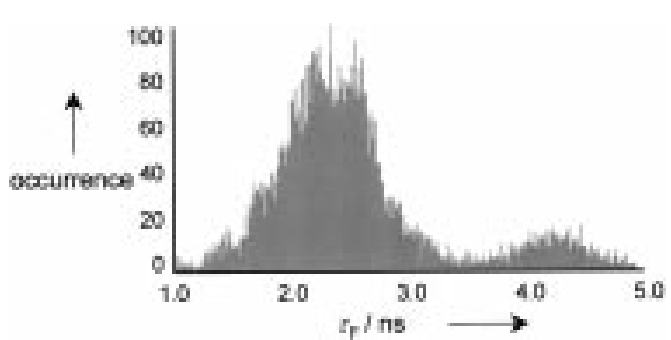

Figure 6. Fluorescence lifetime occurrence containing all the lifetimes recorded in time and space for 68 individual molecules. The broad distribution of $\tau_{F}$ values is partially due to the interaction of the molecules with the nearby dielectric interface as well as to the interaction of the probe molecules with the polymer host.

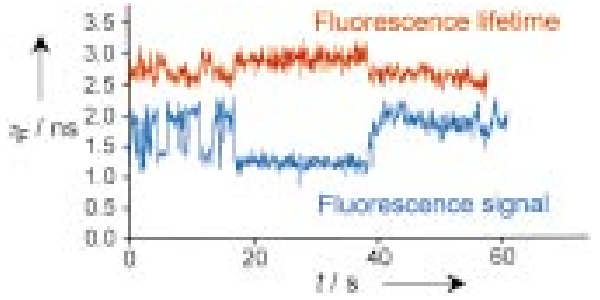

Figure 7. Fluorescence lifetime trajectory (red) and fluorescence intensity time trace (blue) for a particular DiD molecule exhibiting quantum jumps in its lifetime and intensity. For this particular molecule anticorrelation between $\tau_{F}$ and intensity is observed. The anticorrelation might be due to abrupt out-of-plane reorientations of the molecule within the polymer layer.

fluorescence lifetime coincided with a jump in the total intensity. In some cases we have observed correlated jumps, in others anticorrelation was observed. The origin of these jumps is not clear but they might be associated to intrinsic conformational changes in the chromophore itself or external variations of the surrounding, such as chain jumps in the polymer allowing rotational jumps of the probe molecule and/or encounters of the probe molecule with diffusing quenchers. works. Figure 6 shows a fluorescence lifetime distribution containing all the lifetimes recorded in time and space. The histogram can be interpreted as the chance of observing a specific lifetime in a specific place of the sample during a fixed integration time. As clearly seen from the distribution, the lifetime peaks at about $2.4 \mathrm{~ns}$ but the distribution spans from $\sim 1$ up to $4.85 \mathrm{~ns}$. This distribution is much broader than that found by Macklin et al. ${ }^{[13]}$ and cannot be explained solely by orientational effects of the molecules in the presence of a dielectric interface. In fact, we found no anticorrelation between the fluorescence count rate and the fluorescence lifetime measured, an indication that orientation effects are not solely responsible for the broad distribution of the measured lifetimes. On the other hand, it is well known that the relaxation probability of a single molecule is determined by interactions with the neighbouring regions of the polymer. The influence of these interactions on the decay time is dependent on the distance between the molecule and the nearest polymer site. ${ }^{[56]}$ Calculations on an ensemble of molecules interacting with a nonuniform medium have shown deviation of the usual singleexponential behaviour, even in the absence of any quencher. ${ }^{[56]}$ Moreover, the existence of free volume in the polymer facilitates the diffusion of reagent molecules or quenchers. The quencher will contribute with an extra decay channel, thus reducing the fluorescence lifetime. Thus, we believe that such a broad distribution of lifetime values is due to a strong interaction of the probe molecules with the polymer environment. More experiments are of course necessary before drawing final conclusions. In particular, the decay of the probe molecule should be clearly dependent on the polarity of the polymer.

While the majority of the molecules investigated (53 out of 68 molecules) showed rather stable excited-state lifetime behaviour in time, the remaining molecules exhibited some degree of dynamic fluctuation. In most cases, jumps between two or three discrete values of fluorescence lifetime were observed as shown in Figure 7 for a particular molecule. In all cases, a jump in the

\subsubsection{Singlet - Triplet Dynamics}

As mentioned in Section 2.1, besides the repetitive transitions between the ground singlet state $S_{0}$ and the lowest excited singlet state $S_{1}$ giving rise to fluorescence, the molecule has a small chance to undergo intersystem crossing from $S_{1}$ to the lowest excited triplet state $T_{1}$. As long as $T_{1}$ remains occupied, the $\mathrm{S}_{0} \rightarrow \mathrm{S}_{1}$ transition does not occur and the fluorescence is interrupted temporarily. After decaying to $S_{0}$ the molecule starts fluorescing again. Because the lifetime of $T_{1}$ is much longer than that of $\mathrm{S}_{1}$, the fluorescence photons are emitted in bunches separated by dark periods that occur when the molecule is in $T_{1}$. If one could "look" at the real-time trajectory of a single molecule experiencing transitions from $S_{1} \rightarrow S_{0}$ and $T_{1} \rightarrow S_{0}$ one would observe a two-level signal, namely, periods of a continuous fluorescence signal ("on" level), spaced by periods with no signal except from the background ("off").

The first measurements on the $T_{1}$ state of individual molecules were performed at cryogenic temperatures. It was found that the $\mathrm{T}_{1}$ lifetimes and intersystem crossing rates could vary among different molecules, which was attributed to local static disorder in the crystal host. ${ }^{[60,61]}$ At room temperature, single-molecule quantum jumps to $T_{1}$ have been observed using confocal microscopy. ${ }^{[13,18,21,62]}$ Xie and Trautman ${ }^{[19]}$ showed that increasing the imaging time resolution in combination with sufficient signal-to-background ratio allows for real-time observation of ISC to the triplet state. Macklin et al. ${ }^{[13]}$ reported a triplet lifetime of $0.4 \mathrm{~ms}$ for DilC $_{12}$ embedded in PMMA and an intersystem crossing yield of $0.15 \%$ and Yip et al. ${ }^{[21]}$ recently observed triplet state excursions in the ms range of $\mathrm{DilC}_{12}$ adsorbed in PMMA under an $\mathrm{N}_{2}$ atmosphere. $\mathrm{Ha}$ et al. ${ }^{[62]}$ probed intersystem crossing to the triplet state of single Texas Red dye molecules linked to DNA and bound to a glass substrate. From the distribution of values obtained for 40 molecules they found $T_{1}$ lifetimes from 
below 0.02 to $1.3 \mathrm{~ms}$ and a mean ISC yield of $0.2 \%$. Excursions to the $T_{1}$ state of single molecules embedded in different polymer matrices have been also observed in our group ${ }^{[23]}$ using NSOM. The results are summarised below.

Figure 8 a shows a fluorescence time trajectory of a single DilC $_{18}$ molecule embedded in PMMA with a time resolution of $57 \mu \mathrm{s}$ and recorded for $\sim 2 \mathrm{~s}$ until irreversible photobleaching occured using near-field excitation. Figure $8 \mathrm{~b}$ is an expanded view of the same data showing the stochastic character of the on/off switching due to multiple excursions of the molecule to the triplet state. The duration of the dark, "off" intervals is related to the triplet lifetime, and the switching rate is related to the product of the absorption rate and the ISC yield. In the case of a fluorescence quantum yield close to unity, most absorbed photons are re-emitted and the ISC yield can be extracted from the amount of detected photons in the "on" periods. If the
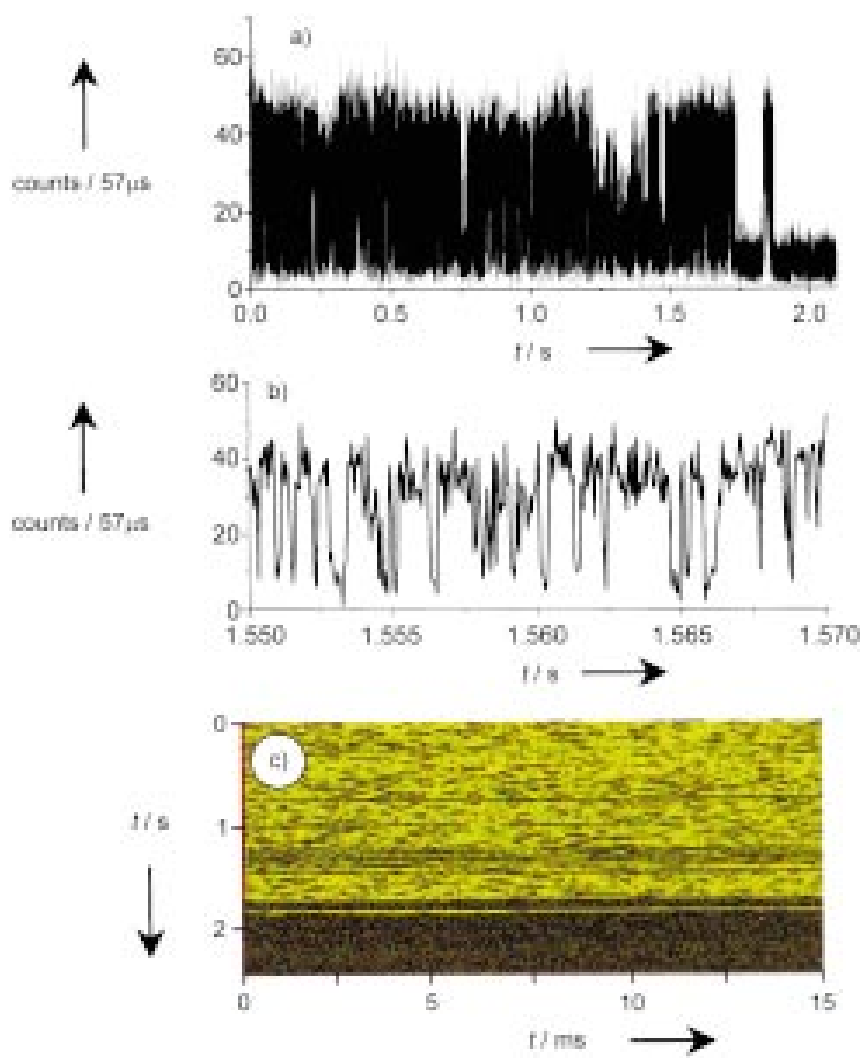

Figure 8. a) Single-molecule time trace with a $57 \mu$ s dwell time. The fluorescence emission rate is $\sim 10^{6}$ count $^{-1}$. Photodissociation of the molecule occurs at $1.85 \mathrm{~s}$, after emission of $2 \times 10^{7}$ photons (set-up collection efficiency of $10 \%$ ). b) Fluorescence time-trace extract over $20 \mathrm{~ms}$. Clearly, the molecule drops its fluorescence emission repeatedly to a low level. This characteristic switching between "on" and "off" (blinking) corresponds to the molecule jumping between singlet and triplet states, respectively. c) A two-dimensional representation of the real time trace shown in (a). Time runs continuously from line to line (left $\rightarrow$ right, top $\rightarrow$ bottom). The bright level represents the photon count rate in a $57 \mu \mathrm{s} \mathrm{bin;}$ bright streaks are due to the $S_{0} \rightarrow S_{1}$ singlet excitation - emission cycle; dark streaks represent the residence in the $T_{1}$ triplet state. Several thousand triplet excursions occur before photodissociation of the molecule. Apart from clearly showing singlet-to-triplet transitions, this two-dimensional representation also serves to indicate the in-plane dipole orientation of the molecule, and clearly shows the existence of dark states, fluorescence intensity fluctuations and abrupt bleaching. excitation intensity is increased, the "on" times are shortened while the "off" times remain the same. As a result, the timeintegrated emission intensity no longer responds linearly to the excitation intensity. This is the most common way in which saturation due to the triplet-state shelving is observed.

Due to the high time resolution involved in the measurements, the conventional graph of fluorescence versus time, such as the one shown in Figure $8 a$, is of little use. The expanded view of Figure $8 \mathrm{~b}$ shows clearly the excursions to the triplet state, albeit in a very limited observation time window. A clearer way of visualising fluorescence trajectories is to plot all data points as colour-scale pixels in a two-dimensional matrix. Figure $8 \mathrm{c}$ shows such a graphical representation, in which time runs horizontally from the upper left pixel, line by line, to the bottom right. The pixel dwell time is $57 \mu$ s and each line is $14 \mathrm{~ms}$. In this way, fluorescence dynamics spanning five orders of magnitude can be displayed in one image. As an additional advantage, the colour coding indicating the polarisation of the emission allows the orientation of the molecule to be monitored.

From each real-time trajectory recorded, statistical values can be determined: the $T_{1}$ state decay time $\tau_{\top}$ and the intersystem crossing yield $Y_{\text {Isc }}$. Figure 9 a shows the distribution of the length of all the dark periods within the observation time for the molecule shown in Figure 8. The distribution displays a monoexponential behaviour, proving that the decay time is characteristic for the lifetime of the dark state, that is, the triplet state lifetime $\tau_{\top}=145 \pm 5 \mu \mathrm{s}$ for this specific molecule. Similarly, the distribution of the number of photons emitted in each light period is plotted in Figure $9 \mathrm{~b}$. Again, the distribution shows a monoexponential behaviour from which an intersystem crossing yield of $2.1 \pm 0.5 \times 10^{-4}$ is estimated. The value of $Y_{\text {ISC }}$ represents the chance per excitation that this specific molecule makes a transition to the triplet state.
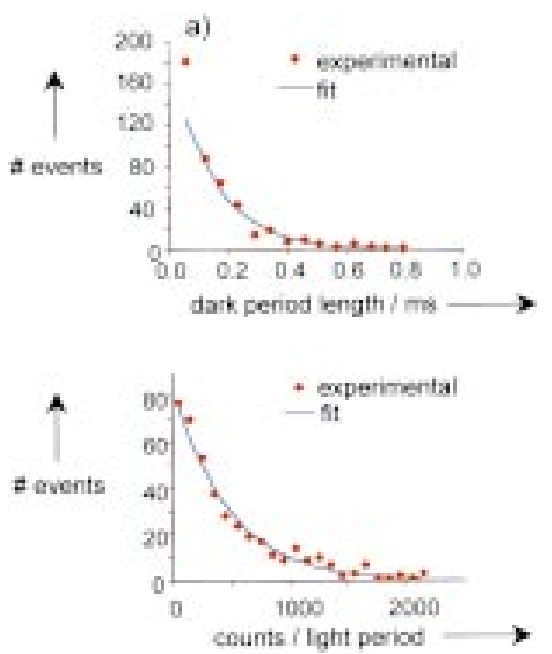

Figure 9. a) Histogram of the length of the "off" periods for the molecule shown in Figure 8. The molecule is embedded in a $10 \mathrm{~nm}$ thick PMMA layer. The single exponential decay corresponds to a mean $T_{1}$ lifetime of $145 \pm 5 \mu \mathrm{s}$ for this particular molecule. b) Histogram of the number of counts between dark periods for the same molecule. A characteristic $\mathrm{Y}_{\text {ISC }}=2.1 \pm 0.5 \times 10^{-4}$ is obtained by fitting the decay with a single exponential. 
The data shown in Figures 8 and 9 correspond to the triplet parameters of only one molecule. In a freely interacting environment, the parameters determined for such a molecule would be the same for all the molecules. However, even at low temperatures and in a crystal host, some degree of disorder exists and as a result the ISC parameters vary from molecule to molecule. ${ }^{[60,61]}$ The situation is much more dramatic at room temperature with molecules embedded in an amorphous polymer. Nevertheless, precisely the variations in the ISC parameters are useful to gain information about the environment. In our experiments we have recorded a large number of fluorescence trajectories of DilC ${ }_{18}$ molecules embedded in ultrathin films of PMMA and PS of similar thickness and have determined the ISC parameters for each molecule in both polymers matrices. As an example, Figure 10 shows the 2D fluorescence trajectories of two different molecules, one embedded in PMMA and the other in PS. Clearly the molecules display very different triplet behaviour. The residence time of the molecule in the triplet state is much longer in PMMA than in PS. Based on the different $\tau_{\top}$ and $Y_{\text {ISC }}$ values obtained for all molecules analysed in both polymers, sets of spatial distributions can be built. Distributions of $\tau_{\top}$ for both polymers render a peak distribution of $\tau_{\top}=170 \mu \mathrm{s}$ in PMMA and $\tau_{\mathrm{T}}=40 \mu \mathrm{s}$ in PS. Similarly, the distributions of $Y_{\text {ISC }}$ in PMMA and PS yield peak values of $3.3 \times 10^{-4}$ and $2.2 \times 10^{-4}$, respectively. ${ }^{[53]}$ In both materials the $\tau_{\top}$ distribution spans more than one order of magnitude and has an asymmetric shape with a tail towards long lifetimes.

The different triplet parameters experienced by the molecules can be understood in terms of oxygen quenching and the heterogeneity of both polymer matrices. Oxygen is known to have two major photophysical effects in most organic dyes. First, it increases the quantum yield of triplet formation by accelerating the rate at which singlet-to-triplet transitions occur due to magnetic interaction. ${ }^{[46,63]}$ Second, oxygen is known to quench the triplet state of the dye molecule via triplet -triplet annihilation with ground-state triplet oxygen. The latter process produces singlet oxygen that can lead to photochemical oxidation. The solubility of oxygen $S$ is about the same in PMMA and PS $\left(8.7 \times 10^{-3}\right.$ and $7.8 \times 10^{-3} \mathrm{molL}^{-1} \mathrm{~atm}^{-1}$, respectively), while the diffusion constant is more than one order of magnitude higher in polystyrene $\left(2.2 \times 10^{-7} \mathrm{~cm}^{2} \mathrm{~s}^{-1}\right.$ in PS and
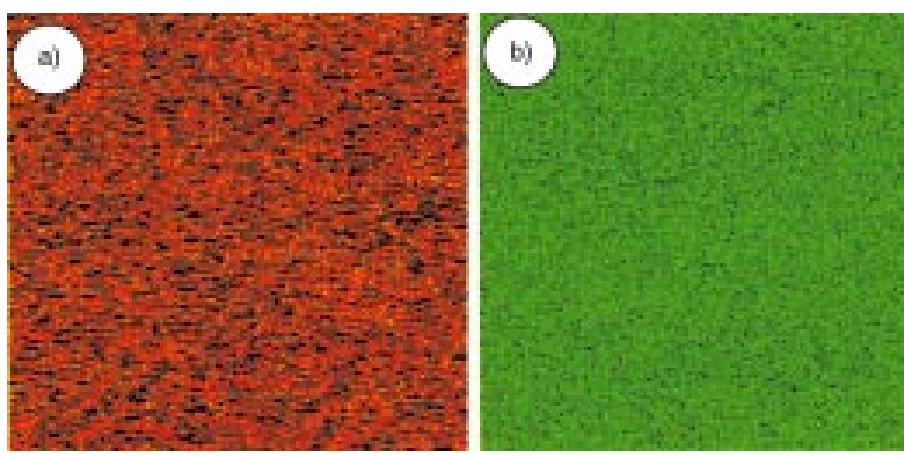

Figure 10. Two-dimensional fluorescence trajectories of two Dil molecules embedded in a) PMMA and b) PS host systems. The pixel dwell time is $57 \mu \mathrm{s}$. Each time trace is $14 \mathrm{~ms}$. Both molecules emit light for $\sim 3.7 \mathrm{~s}$ without photodissociation. The colour in the images reflects the stable in-plane orientation of both molecules. Clearly the dynamics exhibited by both molecules is markedly different, which is a direct proof of the influence of the environment.

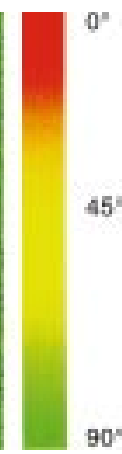

$1.4 \times 10^{-8} \mathrm{~cm}^{2} \mathrm{~s}^{-1}$ in PMMA). ${ }^{[64]}$ On the basis of these parameters, it is reasonable to expect more collisions between the diffusing oxygen and the probe molecule in the PS films. It is therefore not surprising that the triplet lifetimes of the molecules are shorter in PS than in PMMA. A larger diffusion constant of oxygen in polystyrene might be explained in terms of a larger degree of free volume compared to PMMA, which eases the diffusion of small reactive molecules. ${ }^{[46,65]}$ The latter is also consistent with the observation of a larger rotational mobility of the probe molecules in polystyrene films, as discussed in Section 3.1.2. Several groups have confirmed the effect of oxygen on the quenching of the triplet lifetime, not only in ensemble experiments but also at the single-molecular level. ${ }^{[21,66-68]}$ Very recently, the group of Barbara have reported extremely long triplet lifetimes of the same probe molecule in rigorously deoxygenated polymer films $\left(\tau_{\top} \sim 100 \mathrm{~ms}\right)$, with the additional effect of a large increase in the photochemical stability of the probe. ${ }^{[69]}$

While recording fluorescence trajectories, we observed a considerable number of molecules displaying temporal fluctuations of the ISC parameters on a time scale of a few seconds. ${ }^{[23]}$ The temporal distribution of the ISC parameters matched the spatial distribution, satisfying the ergodic principle of statistical physics, stating that for a physically stationary system a time average is equivalent to an ensemble average. We assigned the fluctuations of the triplet parameters to temporal fluctuations in the oxygen concentration in the environment of the probe molecule. ${ }^{[70]}$ More recently, Barbara and co-workers ${ }^{[69]}$ showed that indeed oxygen collisions are the major factor responsible for fluctuations of the intersystem crossing rate in nondegassed and partially degassed polymer films. Furthermore, by comparing fully deoxygenated samples with partially oxygenated samples, the workers were able to establish the degree of static and dynamic disorder in their films.

Clearly, fluorescence lifetime and intersystem crossing parameters with their fully spatial and temporal distributions as obtained by single-molecule analysis can be exploited to thoroughly investigate nanostructure dynamics, heterogeneity and ordering range in polymer systems. In particular, polymer thickness and temperature should have dramatic effects on the photophysical properties of the probe molecules, which in turn will report on the nanostructure, polarity, free volume and transition between surface and bulk behaviour in glassy polymers.

\subsection{Photophysics of Autofluorescent Proteins}

\subsubsection{A Short Look into the Green Fluorescent Protein}

Although biologists are accustomed to and take advantage of organic fluorescence probes for many of their applications, clearly the cloning and expression of the Green Fluorescent Protein (GFP) from the jellyfish Aequorea victoria has revolutionised molecular and cell biology. Since the fluorescence of GFP occurs in the absence of any cofactors, the protein can serve as an exquisite 
marker of gene expression and as a tag for localising proteins within living cells. ${ }^{\left[{ }^{[1,}\right.}{ }^{72]}$ In principle, the DNA sequence coding for GFP can be fused to that of any protein of interest. By expression of the modified gene, the protein is covalently linked to the GFP and thus carries a fluorescent label. ${ }^{[71,72]}$ In the native protein (wild type, wt-GFP) the chromophore is formed in an autocatalytic, posttranslational cyclisation and oxidation of the tripeptide unit at residues $65-67^{[73-75]}$ The wt-GFP exhibits two major absorption bands at $\sim 395$ and $\sim 475 \mathrm{~nm}$, which are associated with protonated and deprotonated states, respectively. Excitation at 395 and/or $475 \mathrm{~nm}$ results in fluorescence at $\sim 508 \mathrm{~nm} \cdot{ }^{[76]}$ Substitution of one or more amino acids at or in close proximity to the chromophore results in mutants with different absorption and emission properties and, in some cases, improved emission and photostability. ${ }^{[74,76,77]}$ A widely used mutant is the S65T-GFP, where the serine at residue 65 is replaced by threonine. ${ }^{[78]}$ In this mutant, the deprotonated state of the chromophore is stabilised and, as a consequence, it shows an absorption peak only at $\sim 475 \mathrm{~nm}$ and a larger absorption cross-section than wtGFP. ${ }^{[72,77,78]}$

Because of the rapidly increasing number of applications, great attention has been focussed on the photophysical properties of the wt-GFP and a number of its mutants. Investigation of the photophysical properties has been carried out in ensemble measurements, at room and low temperatures, ${ }^{[76,79-82]}$ and at the single-molecular level. ${ }^{[83-88]}$ When observed individually, the fluorescence emission of GFP shows intensity fluctuations, on/ off blinking and fluorescence switching, a behaviour that is hidden in ensemble experiments. ${ }^{[84-88]}$ Attempts to clarify the origin of the intensity fluctuations and blinking in the GFP have been reported by several workers. ${ }^{[84-94]}$ Blinking has been reported in all mutants studied so far at the individual molecular level, with time constants and off times spanning from a few microseconds to even hours. The most likely phenomena used to explain the complicated behaviour of the GFP include the existence of protonation states, conformational isomers and triplet states. Fluorescence correlation spectroscopy (FCS) has been used to determine the residence of the protein in the triplet state to be $\sim 30-50 \mu \mathrm{s}^{[89-91]}$ Furthermore, Haupts et al. ${ }^{[91]}$ reported results from measurements of the protonation dynamics of the EGFP and S65T-GFP mutants. At pH values lower than 7 the authors found external protonation of the chromophore from the bulk, whereas at high $\mathrm{pH}$ internal protonation occurred, which the authors associated with conformational rearrangements in the vicinity the chromophore. More recently, Schwille et al. ${ }^{[92]}$ used FCS to observe fast excitation-driven fluctuations in the fluorescence emission of yellow-shifted GFP mutants (T203Y and T203F) on a time scale of $10 \mu$ s to $1 \mathrm{~ms}$. Surprisingly, the fraction of molecules in the dark state remained constant at $\sim 60 \%$ over a wide range of intensities, which indicates that both the population and depopulation of the dark states were light driven. The authors discussed their results in terms of a possible isomerisation of the chromophore as a nonradiative channel of the excited state relaxation. ${ }^{[93]}$ In the earlier work of Dickson et al., ${ }^{[84]}$ autocorrelation analysis from fluorescent trajectories of similar mutants obtained using the total internal reflection excitation at low intensities indicated that the averaged on/off correlation time (in the order of a few seconds) shortened with increased intensity. In contrast, single-molecule studies on the EGFP mutant were reported, using again total internal reflection excitation, with dark periods of $\sim 50$ seconds independent of excitation intensity and on times strongly dependent on the excitation intensity. ${ }^{[87]}$

We applied real-time single-molecule fluorescence detection to study the light-driven dynamics of the S65T-GFP fluorescence emission. ${ }^{[94]}$ In our experiments, we combined confocal microscopy and NSOM to obtain information on the photodynamics of GFP and showed that the excitation intensity has a dramatic effect on GFP blinking, with a reduction of the fraction of molecules in the on state upon increasing excitation intensity. We also found that the on times became shorter at high intensity while the off times were independent of the excitation intensity. In what follows, a brief overview of the results obtained on the S65T-GFP mutant of the GFP is given and compared to that of other workers.

\subsubsection{Imaging Individual Green Fluorescent Proteins}

In order to image spatially and temporally individual GFPs, it is necessary to immobilise the proteins in a physiological environment. We have chosen water-filled pores of poly(acrylamide) (PAA) gels to confine the proteins in space. A polymerisation mixture of acrylamide/bisacrylamide was prepared in phosphate-buffered saline (PBS, pH 7.5). S65T-GFP was included in the mixture to a final concentration of $10^{-7} \mathrm{M}$ for NSOM or $5 \times 10^{-10} \mathrm{M}$ for confocal observation. The acrylamide gels were $15 \%$ by weight with $5 \mathrm{wt}-\%$ crosslinker, yielding average pore diameters of $\sim 3 \mathrm{~nm}$, sufficient to keep the proteins stationary in the gel. A $2 \mu \mathrm{L}$ drop of the GFP/PAA mixture was sandwiched between two silica glass coverslips and the film was allowed to polymerise for a few minutes. The thickness of the resultant gel film was $\sim 3 \mu \mathrm{m}$.

Figure 11 shows a near-field fluorescence image containing individual S65T-GFP proteins. Circularly polarised excitation light

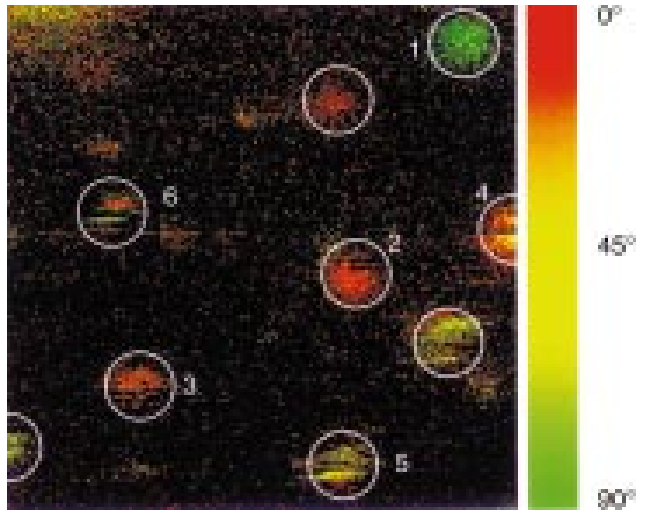

Figure 11. Near-field fluorescence image of individual S65T-GFP molecules embedded in a poly(acrylamide) gel. The scan area is $1.8 \times 1.8 \mu \mathrm{m}^{2}$, with $300 \times$ 300 pixels and acquisition time of $1 \mathrm{~ms}^{\text {pixel }}{ }^{-1}$. Imaging proceeds from top to bottom and left to right. While molecule 1 shows stable emission and stable inplane orientation, molecules 2-4 show some degree of on/off blinking behaviour while maintaining their in-plane orientation. In addition to blinking, molecules 5 and 6 also exhibit some rotational mobility, as clearly seen from the colour changes within one particular fluorescent spot. 
at $\lambda=488 \mathrm{~nm}$ has been used, and the fluorescence emission has been collected using polarisation-sensitive detection. The pseudo-colour coding in the image reflects the relative in-plane orientation of the molecules within the gel matrix. It is important to note that, because of the evanescent character of the light emanating from the near-field aperture probe, only molecules close to the air-gel interface are efficiently excited. A large fraction of the observed GFPs molecules remains stationary in space, as confirmed by the uniform colour of the fluorescent spots. There is however a considerable number of proteins showing some rotational mobility within the gel. This can be observed by the colour changes observed within one individual spot. In principle, each molecule is contained in one of the pores and thus lateral diffusion is greatly inhibited (not observable in our experiments) although the protein can still rotate while embedded in the pore. This rotation is rather slow, since it occurs in the time scale of our experiments $(1 \mathrm{~ms}$ per pixel in this particular image).

More remarkable than rotational activity is, however, the rather noisy behaviour of the GFP as compared to the stability and high signal-to-background obtained on Dil or other organic molecules. The signal-to-background ratio in the image of Figure 11 is typically two, while more than an order of magnitude higher can be routinely obtained in most organic dyes. Increasing the excitation intensity does not improve the fluorescence signal level but, on the contrary, an on/off "blinking" behaviour is clearly apparent when increasing excitation power. The image contains many "stripy" features, a clear signature of the blinking in the GFP. Blinking dynamics and its dependence on excitation intensity is better studied by recording fluorescence time trajectories and determining statistically the duration of the bright and dark periods for a large number of molecules at different excitation conditions.

\subsubsection{Blinking Behaviour and Intensity Dependence}

In an analogous way to the ISC parameters measured on organic molecules, we have also recorded fluorescence time trajectories of individual S65T-GFP proteins at different excitation conditions. Figure 12 shows two time trajectories obtained at two different excitation intensities. The excitation intensity has an clear effect on the blinking behaviour. At low excitation conditions, the emission is rather stable while at high excitation dramatic blinking occurs. In the latter, the fluorescence emission is not continuous in time but it comes as photon bunches spaced by very long dark intervals, much longer that those expected for the triplet-state lifetime of the GFP, on the order of $10-50 \mu$ s. $^{\left[{ }^{[9-91]}\right.}$ In order to extract statistical information of the blinking dynamics of the GFP, we have analysed fluorescence trajectories of a large number of individual S65T-GFP molecules. For each time trajectory we measured a set of times $\Delta t_{\text {on }}$ for the bright intervals and $\Delta t_{\text {off }}$ for the dark intervals. In our analysis $\Delta t_{\text {on }}$ is the time width of a photon burst and $\Delta t_{\text {off }}$ is defined as the time between two consecutive photon bursts, including those dark times present between the start of the experiment and the first fluorescent burst. Figure $12 \mathrm{c}$ shows the $\Delta t_{\text {off }}$ distribution for a set of 65 molecules. The histogram is fitted with a single exponential a)
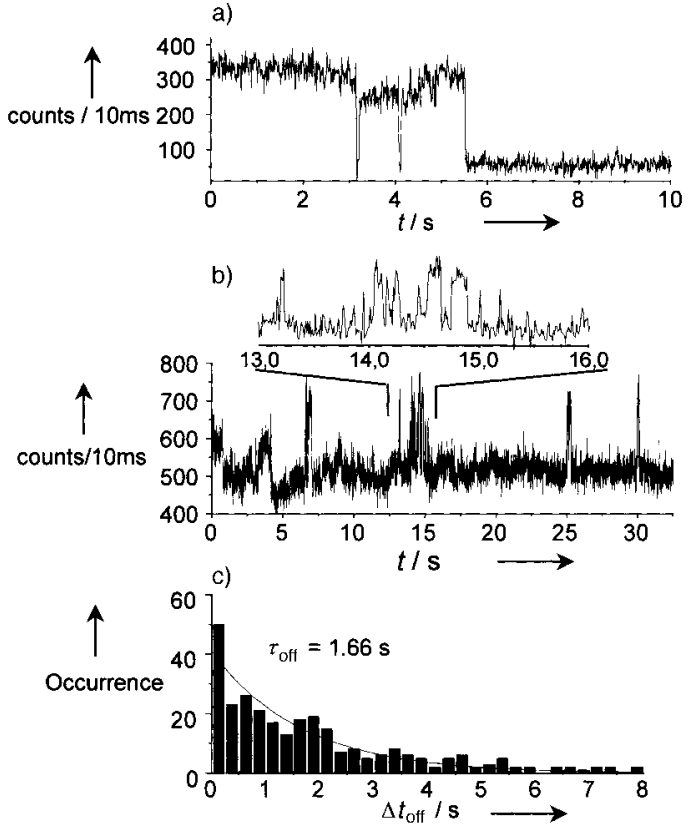

Figure 12. Real-time fluorescence trajectories of two different S65T-GFP molecules embedded in a PAA gel. The excitation intensities were a) 1.2 and b) $14 \mathrm{~kW} \mathrm{~cm}^{-2}$. Note that in (a) the emission is essentially constant except from the two rather short dark intervals occurring before photodissociation. In (b), the fluorescence emission is interrupted by long dark intervals. The inset shows a time extract of $\sim 3 \mathrm{~s}$, which exhibits blinking on a time scale of $\sim 0.5 \mathrm{~s}$. c) Histogram of the length of the dark, off periods obtained for 64 proteins observed continuously during $\sim 60$ s excitation at $\sim 14 \mathrm{~kW} \mathrm{~cm}^{-2}$. The monoexponential decay of the dark period distribution corresponds to $\tau_{\text {off }}=1.6 \pm 0.2 \mathrm{~s}$.

decay with a characteristic decay time $\tau_{\text {off }}=1.6 \pm 0.2 \mathrm{~s}$. With increased excitation intensity the duration the dark intervals remained constant, whereas the $\Delta t_{\text {on }}$ values reduced dramatically and the number of photon counts contained in each photon burst increased linearly. ${ }^{[94]}$

The observed single-molecule photodynamics of the S65TGFP mutant revealed the existence of a long-lived dark state. We can understand this behaviour if we model the GFP as a threelevel system, where transitions from the singlet excited and ground states result in the emission of photons. For lowexcitation conditions, the number of emitted photons is proportional to the excitation intensity. There is however a small probability that while being in the excited state, the molecule makes a transition to the long-lived dark state $D$. During the time interval the molecule is in $D$ there is no fluorescence emission and the resultant signal appears as off blinking. Only when the molecule returns to the singlet ground state and absorbs a new photon, fluorescence will resume. The number of transitions to the dark state per second increases with increasing excitation intensity. The transition probability from a bright to a dark state is given by the inverse of the average number of emitted photons during the on state, while the residence time in $D$ will be the off-blinking time.

At present, the exact nature of the $D$ state is not known. Because of the time scale of the dark periods found in our experiments, we exclude the possibility that the $D$ state would be a triplet state of the GFP. Recent quantum chemical 
calculations by Weber et al. ${ }^{[93]}$ support the notion that an additional zwitterionic $Z$ form of the chromophore exists in the wt-GFP and a number of its mutants. The $Z$ form would be responsible for the interruption of fluorescence observed in single-molecule experiments. Recent experimental data from Creemers et al. ${ }^{[81,82]}$ revealed the existence of at least three photointerconvertible forms of the wt-GFP, also present in redshifted mutants, including the S65T-GFP. Photointerconversions between different forms would correspond to the on/off blinking observed at room temperature. To test the role of interconversion between different forms of the chromophore, Jung et al. ${ }^{[88]}$ have recently conducted room temperature, twocolour fluorescence excitation spectroscopy on two different GFP mutants. FCS of a small number of molecules clearly showed photoconversion between the anionic and neutral forms of the chromophore in the case of the E222Q mutant but showed little effect in the EGFP mutant (F64L/S65T), the latter being very similar to the S65T-GFP used in our experiments. Most probably, a number of different processes, including fast internal conversion, proton exchange and photoconversion between different states, are, amongst others, contributing simultaneously to the complex photophysical behaviour displayed by the protein. It is remarkable to notice that a large number of papers have been published so far, using different techniques from bulk to single-molecule sensitivity, reporting the existence of GFP blinking at rather different time scales (from the nanoseconds when using $\mathrm{FCS}^{[88-92]}$ to many seconds when using wide-field illumination $\left.{ }^{[87]}\right)$. A comprehensive review on single-molecule spectroscopy of the GFP, including comparison of optical detection techniques, mutants and different on/off blinking time scales has been published recently. ${ }^{[95]}$

\subsubsection{Photobleaching and Blinking}

In addition to the on/off times, from the fluorescence trajectories one can also extract the probability of photobunching occurrence in time. Figure 13 shows the probability of finding a bunch

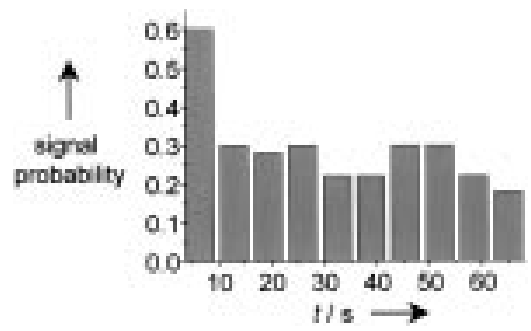

Figure 13. Photon bunch probability occurrence in time. The distribution has been built over 86 molecules by recording the occurrence time of a photon bunch during the observation time in which each molecule is continuously illuminated at $\lambda=488 \mathrm{~nm}$ and excitation intensity $14 \mathrm{~kW} \mathrm{~cm}^{-2}$. A typical organic molecule will photodissociate after a few seconds of excitation, whereas in the case of the GFP there is a nonnegligible probability that the protein will emit a bunch of photons after $60 \mathrm{~s}$ of excitation. In an ensemble measurement, where many proteins will fluorescence intermittently in a asynchronous fashion, an average fluorescence emission will be observed for an extraordinary long illumination time and mistakenly interpreted as a very stable emission and resistance of the GFP to photobleaching. of photons during the observation time. Although the distribution clearly shows that within the first few seconds after illumination the probability to observe photon emission is highest, the distribution remains rather flat up to 65 seconds of continuous irradiation. The total number of photons emitted by each molecule during the observation time is approximately $2.5 \times 10^{5}$ photons, which indicates that the photodissociation rate of the protein is $<4 \times 10^{-6}$. These numbers are rather similar to those obtained on most organic molecules. The main difference is the required time to collect that number of photons from a GFP molecule. This might explain ensemble observations on the excellent stability of the protein against photobleaching. ${ }^{[7]}$ In an ensemble experiment there will always be a number of proteins in an on state, delivering enough fluorescence, while the large majority will be in a dark, nonemissive state, "saving" their photons. Only after emission of $\sim 10^{5}$ photons will the chromophore photodissociate, which, in the case of the GFP, it will take many seconds or even minutes at high excitation intensities. Figure 13 reflects once more the power of singlemolecule experiments as to compared to the equivalent ensemble photobleaching experiment. Finally, it is also important to mention that out of 86 molecules investigated in our experiments, only 64 returned from a dark state within the observation time making statistical analysis of $\Delta t_{\text {on }}$ and $\Delta t_{\text {off }}$ possible. The remaining $22(26 \%)$ either photodissociated or remained dark for a period longer than $65 \mathrm{~s}$. Actually, Peterman et al. ${ }^{[87]}$ has reported recently single-molecule studies on the EGFP mutant with dark periods of $\sim 50$ seconds, independent of excitation intensity. The same workers discussed the recovery of fluorescence emission after long dark periods, also considered as reversible photobleaching. In the case of the EGFP mutant, the restoration of the emissive form occurred spontaneously and it was not light driven, ${ }^{[87]}$ while for yellow GFP mutants such as the T203Y and the E222Q, recovery of emission was achieved after illumination with $405 \mathrm{~nm}$ light. ${ }^{[84,88]}$ In a series of elegant twocolour FCS experiments, Jung et al. ${ }^{[88]}$ showed a large increase of fluorescence emission on the E222Q mutant by simultaneous excitation with 476 and $407 \mathrm{~nm}$ light. The workers explained the increase in fluorescence signal as an efficient repopulation of the dark state involving a neutral chromophore which is only produced upon $476 \mathrm{~nm}$ illumination. Certainly many more experiments need to be performed to unravel the complicated photophysics of the GFP and its mutants, in particular the relation between blinking and (ir)reversible photobleaching.

\section{Conclusions, Prospects and Future Directions}

Single-molecule detection has become, in only a few years, an established research area with widespread impact in pure and applied sciences. Initially, most of the single-molecule optical studies were largely phenomenological in nature: measurement of photophysical properties at the individual level which often revealed new and unexpected effects. However, the technique is now becoming sufficiently well established that it can be employed not only to seek new phenomena but also to provide 
rigorous tests of previously developed models. A few examples have been discussed in this Minireview.

We have shown that single molecules can be used as exquisite probes to investigate polymer dynamics, microstructure and nanoheterogeneity of different polymer matrices. We have performed fast time-resolved experiments in the fluorescence emission of single molecules revealing variations on the fluorescence lifetimes of nominally identical molecules. We have also observed real-time single molecule excursions to the triplet state. A broad distribution of the ISC parameters has been found, reflecting the heterogeneity of the molecular environment. The dynamic behaviour of the triplet parameters is dependent on the polymer host and the oxygen content in the polymer, demonstrating that both oxygen concentration variations and conformational changes mediated by the environment play a role in the triplet-state dynamics. In some cases, we have seen correlation between triplet-state dynamics and changes in the quantum emission yield and rotational activity. As a whole, these results demonstrate that monitoring the photodynamics and orientation of individual guest molecules in a polymer host provides a new and direct way to probe polymer dynamics at the molecular scale.

We have also described the photodynamics exhibited by the green fluorescence protein. Several groups using single-molecule approaches are investigating the extremely complex photophysical behaviour of this interesting protein. Unexpected phenomena such as blinking are now being unmasked, and controversial and/or irreproducible results obtained in ensemble experiments might be understood on the basis of the singlemolecule data.

Recent advances in room temperature single-molecule studies have occurred at an extremely rapid pace, often in a surprising manner, to generate exciting possibilities in many disciplines. Single-molecular reorientation dynamics has enormous potential to elucidate structural behaviour at the molecular scale for a variety of systems including polymers, dendritic molecules, artificial and biological membranes, and proteins. Single-pair fluorescence energy transfer (spFRET) is a new and powerful technique, capable not only of exquisite spatial colocalisation but also used to report on dynamic changes in the distance or orientation between two individual chromophores. $^{[29,30]}$ Different groups are already using spFRET to measure intra- and/or intermolecular conformational changes of a number of different proteins and enzymes while in their natural environment.

Future single-molecule experiments will be more successful once different techniques are combined. Already the group of Yanagida has reported examples of single-molecule experiments combining fluorescence detection at the total internal reflection geometry, together with force sensing in an optical trap and a scanning nanoneedle probe. ${ }^{[96]}$ A general trend can be recognised towards the experimental combination of different techniques for single-molecule detection, imaging, spectroscopy and manipulation. Such increasing experimental diversity is required in order to tackle the many unresolved questions on the behaviour of matter on the molecular scale. While the combination of different techniques is certainly important, clearly the future in the single-molecule field will rely on the interdisciplinary approach of physical, chemical and biological sciences.

A large number of people have contributed to the work described here. We would like to thank T. Ruiter, N. van der Berg, K. van der Werf, J. Korterik, F. Segerink, J. van Noort, W. Rensen, I. Segers-Nolten, B. de Bakker, M. Koopman, B de Grooth, L. Kuipers and J. Vancso for assistance and fruitful discussions. We are also grateful to V. Subramaniam and T. M. Jovin in the Max Plank Institute for Biophysical Chemistry, Göttingen, for purified S65TGFPs. The research of M.F.G.-P. has been made possible by a fellowship of the Royal Netherlands Academy of Arts and Sciences (KNAW). J.-A.V. is supported by the Netherlands Foundation for Fundamental Research of Matter (FOM). The work of R.V. is supported by the Netherlands Foundation of Chemical Research (CW).

[1] A. Janshoff, M. Neitzert, Y. Oberdörfer, H. Fuchs, Angew. Chem. 2000, 112, 3346; Angew. Chem. Int. Ed. 2000, 39, 3212.

[2] J. K. Gimzewski, C. Joachim, Science 1999, 283, 1683.

[3] E. Betzig, R. J. Chichester, Science 1993, 262, 1422.

[4] Th. Schmidt, G. J. Schütz, W. Baumgartner, H. J. Gruber, H. Schindler, J. Phys. Chem. 1995, 99, 17662.

[5] R. M. Dickson, D. J. Norris, Y. L. Tzeng, W. E. Moerner, Science 1996, 274, 966.

[6] M. A. Bopp, A. J. Meixner, G. Tarrach, I. Zschokke-Gränacher, L. Novotny, Chem. Phys. Lett. 1996, 263, 721.

[7] A. G. T. Ruiter, J. A. Veerman, M. F. García-Parajó, N. F. van Hulst, J. Phys. Chem. A 1997, 101, 7318.

[8] X. N. Xu, E. S. Yeung, Science 1997, 275, 1106.

[9] T. Ha, T. Enderle, D. S. Chemla, P. R. Selvin, S. Weiss, Phys. Rev. Lett. 1996, 77, 3979.

[10] T. Ha, J. Glass, T. Enderle, D. S. Chemla, S. Weiss, Phys. Rev. Lett. 1998, 80, 2093.

[11] T. Ha, T. A. Laurence, D. S. Chemla, S. Weiss, J. Phys. Chem. B 1999, 103, 6839.

[12] J. K. Trautman, J. J. Macklin, L. E. Brus, E. Betzig, Nature 1994, 369, 40.

[13] J. J. Macklin, J. K. Trautman, T. D. Harris, L. E. Brus, Science 1996, 272, 255.

[14] J. K. Trautman, J. J. Macklin, Chem. Phys. 1996, 205, 221.

[15] X. S. Xie, Acc. Chem. Res. 1996, 29, 598.

[16] H. P. Lu, X. S. Xie, Nature 1997, 385, 143.

[17] H. P. Lu, L. Xun, X. S. Xie, Science 1998, 282, 1877.

[18] Single Molecule Detection, Imaging, and Spectroscopy (Eds.: T. Basché, W. E. Moerner, M. Orrit, U. P. Wild), VCH, Weinheim, 1997.

[19] X. S. Xie, J. K. Trautman, Annu. Rev. Phys. Chem. 1998, 49, 441.

[20] W. E. Moerner, M. Orrit, Science 1999, 283, 1670.

[21] W.-T. Yip, D. Hu, J. Yu, D. A. Vanden Bout, P. F. Barbara, J. Phys. Chem. A 1998, 102, 7564

[22] D. A. Vanden Bout, W.-T. Yip, D. Hu, D.-K. Fu, T. M. Swager, P. F. Barbara, Science 1997, 277, 1074.

[23] J. A. Veerman, M. F. García-Parajó, L. Kuipers, N. F. van Hulst, Phys. Rev. Lett. 1999, 83, 2155.

[24] M. Sauer, K. H. Drexhage, U. Lieberwirth, R. Müller, S. Nord, C. Zander, Chem. Phys. Lett. 1998, 284, 153.

[25] Y. Harada, T. Funatsu, K. Murakami, Y. Nonoyama, A. Ishihama, T. Yanagida, Biophys. J. 1999, 76, 709.

[26] L. Edman, U. Mets, R. Rigler, Proc. Natl. Acad. Sci. USA 1996, 93, 6710.

[27] Y. Jia, A. Sytnik, L. Li, S. Vladimirov, B. S. Cooperman, R. M. Hochstrasser, Proc. Natl. Acad. Sci. USA 1997, 94, 7932.

[28] T. Ha, T. Enderle, D. F. Ogletree, D. S. Chemla, P. R. Selvin, S. Weiss, Proc. Natl. Acad. Sci. USA 1996, 93, 6264.

[29] S. Weiss, Science 1999, 283, 1676.

[30] S. Weiss, Nat. Struct. Biol. 2000, 7, 724.

[31] Y. Ishii, T. Yanagida, Single Mol. 2000, 1, 5. 
[32] J. Yu, D. Hu, P. F. Barbara, Science 2000, 289, 1327.

[33] T. Huser, M. Yan, L. J. Rothberg, Proc. Natl. Acad. Sci. USA 2000, 97, 11187.

[34] J. Hofkens, M. Maus, T. Gensch, T. Vosch, M. Cotlet, F. Köhn, A. Herrmann, K. Müllen, F. De Schryver, J. Am. Chem. Soc. 2000, 122, 9278.

[35] J. R. Lakowicz, Principles of Fluorescence Spectroscopy, Plenum, New York, NY, 1983.

[36] J. Mertz, C. Xu, W. W. Webb, Opt. Lett. 1995, 20, 2532.

[37] E. J. Sanchez, L. Novotny, G. R. Holtom, X. S. Xie, J. Phys. Chem. 1997, 101, 7019.

[38] C. Eggeling, L. Brand, C. A. M. Seidel, Bioimaging 1997, 5, 105.

[39] H. Yokota, K. Saito, T. Yanagida, Phys. Rev. Lett. 1998, 80, 4606.

[40] S. Nie, S. R. Emory, Science 1997, 275, 1102.

[41] K. Kneipp, Y. Wang, H. Kneipp, L. T. Perelman, I. Itzkan, R. R. Dasari, M. S. Field, Phys. Rev. Lett. 1997, 78, 1667.

[42] L. H. Sterling, Introduction to Physical Polymer Science, 2nd ed., Wiley, New York, NY, 1992.

[43] P. J. Flory, Principles of Polymer Chemistry, Cornell University Press, Ithaca, NY, 1953.

[44] V. P. Privalko, Y. S. Lipatov, Makromol. Chem. 1972, 175, 641.

[45] W. Pechnold, IUPAC Prepr. 1971, 789.

[46] J. E. Guillet, Polymer Photophysics and Photochemistry: An Introduction to the Study of Photoprocesses in Macromolecules, Cambridge University Press, Cambridge, 1985.

[47] E. A. Prado, S. B. Yamaki, T. D. Z. Atvars, O. E. Zimerman, R. G. Weiss, J. Phys. Chem. $B$ 2000, 104, 5905, and references therein.

[48] J. A. Veerman, M. F. García-Parajó, L. Kuipers, N. F. van Hulst, J. Microsc. $1999,194,477$.

[49] N. F. van Hulst, J. A. Veerman, M. F. García-Parajó, L. Kuipers, J. Chem. Phys. 2000, 112, 7799

[50] G. S. Harms, M. Sonnleitner, G. J. Schütz, H. J. Gruber, T. Schmidt, Biophys. J. 1999, 77, 2864.

[51] T. Kajiyama, K. Tanaka, A. Takahara, Macromolecules 1997, 30, 280

[52] R. A. Jones, R. W. Richards, Polymers at Surfaces and Interfaces, Cambridge University Press, Cambridge, 1999, p. 53.

[53] "Single-Molecule Detection with a Near-Field Optical Microscope": J. A. Veerman, Ph.D. thesis, University of Twente (The Netherlands), 1999.

[54] A.M. North, Molecular Behaviour and the Development of Polymer Materials, Chapman and Hall, London, 1975.

[55] K. Sienicki, S. Blonski, G. Durocher, J. Phys. Chem. 1991, 95, 1576.

[56] S. Draxler, M. E. Lippitsch, I. Klimant, H. Kraus, O. S. Wolfbeis, J. Phys. Chem. $1995,99,3162$

[57] X. S. Xie, R. C. Dunn, Science 1994, 265, 361

[58] W. P. Ambrose, P. M. Goodwin, J. C. Martin, R. A. Keller, Science 1994, 265, 364.

[59] P. Tinnefeld, V. Buschmann, D.-P. Herten, K.-T. Han, M. Sauer, Single Mol. 2000, 1, 215.

[60] M. Orrit, J. Bernard, Phys. Rev. Lett. 1990, 65, 2716.

[61] T. Basché, S. Kummer, C. Bräuchle, Nature 1995, 373, 132.

[62] T. Ha, T. Enderle, D. S. Chemla, P. R. Selvin, S. Weiss, Chem. Phys. Lett. 1997, $271,1$.

[63] N. J. Turro, Modern Molecular Photochemistry, University Science Books, Mill Valley, CA, 1991.

[64] J. M. Charlesworth, T. H. Gan, J. Phys. Chem. 1996, 100, 14922.

[65] R. N. Haward, R.J. Young, The Physics of Glassy Polymers, 2nd ed., Chapman \& Hall, London, 1997, p. 110
[66] K. D. Weston, S. K. Buratto, J. Phys. Chem. A 1998, 102, 3635.

[67] K. D. Weston, P. J. Carson, J. A. DeAro, S. K. Buratto, Chem. Phys. Lett. 1999, $308,58$.

[68] K. D. Weston, P. J. Carson, H. Metiu, S. K. Buratto, J. Chem. Phys. 1998, 109, 7474.

[69] D. S. English, A. Furube, P. F. Barbara, Chem. Phys. Lett. 2000, 324, 15.

[70] K. Schmidt-Rohr, A. S. Kulik, H. W. Beckham, A. Ohlemacher, U. Pawelzik, C. Boeffel, H. W. Spiess, Macromolecules 1994, 27, 4733.

[71] Green Fluorescent Protein: Properties, Applications and Protocols (Eds.: M. Chalfie, S. Kain), Wiley, New York, NY, 1998.

[72] R. Y. Tsien, Annu. Rev. Biochem. 1998, 67, 509.

[73] M. Chalfie, Y. Tu, G. Euskirchen, W. W. Ward, D. C. Prasher, Science 1994, $263,802$.

[74] R. Heim, D. C. Prasher, R. Y. Tsien, Proc. Natl. Acad. Sci. USA 1994, 91, 12501.

[75] B. G. Reid, G. C. Flynn, Biochemistry 1997, 36, 6786.

[76] M. Chattoraj, B. A. King, G. U. Bublitz, S. G. Boxer, Proc. Natl. Acad. Sci. USA $1996,93,8362$

[77] A. B. Cubitt, R. Heim, S. R. Adams, A. E. Boyd, L. A. Gross, R. Y. Tsien, Trends Biochem. Sci. 1995, 20, 448

[78] K. Brejc, T. K. Sixma, P. A. Kitts, S. R. Kain, R. Y. Tsien, M. Ormö, S. J. Remington, Proc. Natl. Acad. Sci. USA 1997, 94, 2306.

[79] H. Lossau, A. Kummer, R. Heinecke, F. Pöllinger-Dammer, C. Kompa, T. Jonsson, C. M. Silva, M. M. Yang, D. C. Youvan, M.-E. Michel-Beyerle, Chem. Phys. 1996, 213, 1.

[80] A. Kummer, C. Kompa, H. Lossau, F. Pöllinger-Dammer, M. E. MichelBeyerle, C. M. Silva, E. J. Bylina, W. J. Coleman, M. M. Yang, D. C. Youvan, Chem. Phys. 1998, 237, 183.

[81] T. M. H. Creemers, A. J. Lock, V. Subramaniam, T. M. Jovin, S. Völker, Nat. Struct. Biol. 1999 6, 557.

[82] T. M. H. Creemers, A. J. Lock, V. Subramaniam, T. M. Jovin, S. Völker, Proc. Natl. Acad. Sci. USA 2000, 97, 2974.

[83] D. W. Pierce, N. Hom-Booher, R. D. Vale, Nature 1997, 388, 338.

[84] R. M. Dickson, A. B. Cubitt, R. Y. Tsien, W. E. Moerner, Nature 1997, 388, 355

[85] W. E. Moerner, E. J. G. Peterman, S. Brasselet, S. Kummer, R. M. Dickson, Cytometry 1999, 36, 232.

[86] M. F. García-Parajó, J.-A. Veerman, G. M. J. Segers-Nolten, B. G. de Grooth, J. Greve, N. F. van Hulst, Cytometry 1999, 36, 239.

[87] E. J. G. Peterman, S. Brasselet, W. E. Moerner, J. Phys. Chem. A 1999, 103, 10553.

[88] G. Jung, S. Mais, A. Zumbusch, C. Bräuchle, J. Phys. Chem. A 2000, 104, 873.

[89] A. J. W. G. Visser, M. A. Hink, J. Fluoresc. 1999, 9, 81.

[90] J. Widengren, U. Mets, R. Rigler, Chem. Phys. 1999, 250, 171.

[91] U. Haupts, S. Maiti, P. Schwille, W. W. Webb, Proc. Natl. Acad. Sci. USA 1998, 95, 13573.

[92] P. Schwille, S. Kummer, A. A. Heikal, W. E. Moerner, W. W. Webb, Proc. Natl. Acad. Sci. USA 2000, 97, 151.

[93] W. Weber, V. Helms, J. A. McCammon, P. W. Langhoff, Proc. Natl. Acad. Sci. USA 1999, 96, 6177

[94] M. F. García-Parajó, G. M. J. Segers-Nolten, J.-A. Veerman, J. Greve, N. F. van Hulst, Proc. Natl. Acad. Sci. USA 2000, 97, 7237.

[95] A. Zumbusch, G. Jung, Single Mol. 2000, 1, 261.

[96] K. Kitamura, M. Tokunaga, A. H. Iwane, T. Yanagida, Nature 1999, 397, 129.

Received: December 11, 2000 [M 162] 